OPEN ACCESS

Edited by:

Yujun Shi,

Sichuan University, China

Reviewed by:

Fei Wu Long

Sichuan University, China

Michele Ghidini,

IRCCS Foundation Ca 'Granda

Ospedale Maggiore Policlinico, Italy

*Correspondence:

Zhibo Zhao

zhaozhibo@stu.cqmu.edu.cn

Lei Zhao

zhaolei@cqmu.edu.cn

Specialty section:

This article was submitted to Gastrointestinal Cancers,

a section of the journal

Frontiers in Oncology

Received: 11 February 2021

Accepted: 26 April 2021

Published: 14 May 2021

Citation:

Lin Z, Gong J, Zhong G, Hu J,

Cai D, Zhao $L$ and Zhao Z (2021)

Identification of Mutator-Derived Alternative Splicing Signatures of

Genomic Instability for Improving

the Clinical Outcome of

Cholangiocarcinoma

Front. Oncol. 11:666847.

doi: 10.3389/fonc.2021.666847

\section{Identification of Mutator-Derived Alternative Splicing Signatures of Genomic Instability for Improving the Clinical Outcome of Cholangiocarcinoma}

\author{
Zijing Lin ${ }^{1}$, Jianping Gong ${ }^{2}$, Guochao Zhong ${ }^{2}$, Jiejun $\mathrm{Hu}^{2}$, Dong $\mathrm{Cai}^{2}$, Lei Zhao ${ }^{3 *}$ \\ and Zhibo Zhao ${ }^{2 *}$ \\ ${ }^{1}$ Department of Breast and Thyroid Surgery, the Second Affiliated Hospital of Chongqing Medical University, \\ Chongqing, China, ${ }^{2}$ Department of Hepatobiliary Surgery, The Second Affiliated Hospital, Chongqing Medical University, \\ Chongqing, China, ${ }^{3}$ Department of Hepatobiliary Surgery, The Second Affiliated Hospital \& Centre for Lipid Research \& Key \\ Laboratory of Molecular Biology for Infectious Diseases (Ministry of Education), Chongqing Medical University, \\ Chongqing, China
}

Background: Cholangiocarcinoma is an aggressive carcinoma with increasing incidence and poor outcomes worldwide. Genomic instability and alternative splicing (AS) events are hallmarks of carcinoma development and progression. The relationship between genomic instability, AS events, and tumor immune microenvironment remain unclear.

Methods: The splicing profiles of patients with cholangiocarcinoma were obtained from The Cancer Genome Atlas (TCGA) spliceSeq database. The transcriptomics, simple nucleotide variation (SNP) and clinical data of patients with cholangiocarcinoma were obtained from TCGA database. Patients were divided into genomic unstable (GU-like) and genomic stable (GS-like) groups according to their somatic mutations. Survival-related differential AS events were identified through integrated analysis of splicing profiling and clinical data. Kyoto Encyclopedia of Genes and Genomes enrichment analysis was used to identify AS events occurring in genes enriched in cancer pathways. Pearson correlation was applied to analyze the splicing factors regulating AS events. CIBERSORT was used identify differentially infiltrating immune cells.

Results: A prognostic signature was constructed with six AS events. Using this signature, the hazard ratio of risk score for overall survival is 2.362. For TCGA patients with cholangiocarcinoma, the area under the receiver operating characteristic curve is 0.981. CDK11A is a negative regulator of survival associated AS events. Additionally, the CD8+ T cell proportion and PD-L1 expression are upregulated in patients with cholangiocarcinoma and high splicing signatures. 


\begin{abstract}
Conclusion: We provide a prognostic signature for cholangiocarcinoma overall survival. The CDK11A splicing factor and SLC46A1-39899-ES and IARS-86836-ES AS events may be potential targets for cholangiocarcinoma therapy. Patients with high AS risk score may be more sensitive to anti-PD-L1/PD1 immunotherapy.
\end{abstract}

Keywords: cholangiocarcinoma, genomic instability, alternative splicing, immunotherapy, overall survival

\section{INTRODUCTION}

Cholangiocarcinoma describes a group of carcinomas that occur in the biliary tree. Cholangiocarcinoma accounts for approximately $15 \%$ of all primary liver tumors and $3 \%$ of gastrointestinal cancers and the incidence of cholangiocarcinoma is increasing globally (1). In early stages cholangiocarcinoma is asymptomatic, leading to diagnosis in advanced stages and poor patient prognosis (2). The 5year survival rate for patients with cholangiocarcinoma is $7-20 \%$ and tumor recurrence rates after resection remain disappointing (3). Therefore, there is an urgent need to find new biomarkers for cholangiocarcinoma diagnosis and prognosis.

Genomic instability is a driving factor of caner (4), and is associated with poor outcome in patients with cholangiocarcinoma $(5,6)$. To date, the molecular mechanisms of genomic instability in cholangiocarcinoma remain unclear. Recently, some microRNA (miRNA) 48 and long non-coding RNA (lncRNA) signatures associated with genomic instability have been identified. These signatures have efficiently predicted the outcome of ovarian cancer and breast carcinoma (7). However, whether alternative splicing (AS) events are associated with genomic instability remains unclear. However, whether genomic instability-related alternative splicing events predicted the outcome of cholangiocarcinoma remains unclear.

AS is a process through which exons within the same gene are expressed in different combinations, allowing a single gene to produce different proteins at different times and in different environments (8). The unbalanced expression of different isoforms of a single gene is recognized as contributing to the tumorigenesis and progression of numerous carcinomas (9). CD44v8-10 isoforms are upregulated in cholangiocarcinoma, and promote the proliferation of cholangiocarcinoma cells (10). Similarly, AS alternative events in WISP1v, Nek2B, $\Delta$ EX2TFF2, Foxp3 $\Delta 3, \Delta 133$ p53, PKM2, EP3-4, and AGR2vH are associated with the proliferation, migration, and invasion of cholangiocarcinoma cells (11).

In addition to affecting tumor cells, AS affects immune cells in the tumor microenvironment. Unbalanced ESRI1 isoforms are linked with infiltrating lymphocyte activity and patient survival (12). Similarly, AS events have been evaluated as predictive biomarkers for tumor immunotherapy in gastric cancer and squamous cell carcinoma $(13,14)$. Therefore, dysregulated AS events may serve as prognosis indicators and as potential therapy targets.

In this study, we describe a new prognostic signature model based on genomic instability derived AS events. Additionally, we explore the splicing factors that regulate the alternative splicing events recruited in our model. Furthermore, we analyzed the infiltrating immune cells correlated with this prognostic signature.

\section{METHODS}

\section{Data Collection}

Transcriptomics, simple nucleotide variation, and clinical phenotype data of patients with cholangiocarcinoma $(n=36)$ were downloaded from The Cancer Genome Atlas (TCGA) database (https://tcga-data.nci.nih.gov/). AS data of patients with cholangiocarcinoma $(\mathrm{n}=36)$ were downloaded from the TCGAspliceSeq database (http://bioinformatics.mdanderson. org). Percent splicing index values for AS events were applied to reflect the likelihood of each AS event.

\section{Identification of Genomic Instability Associated AS Events}

To identify genomic instability associated AS events, a mutator hypothesis-derived tumor genome computational framework combining Percent Spliced In (PSI) values of AS events and somatic mutation profiles was developed (Figure 1). This framework involved calculating the cumulative quantity of somatic mutations for each patient, and ranking patients in descending order of somatic mutation quantity. Then, the top $25 \%(n=9)$ and the last $25 \%(n=9)$ of patients were defined as genomic unstable (GU-like) and genomic stable (GS-like) group respectively. PSI values of AS events were compared between GUlike and GS-like groups with significance analysis of microarrays method. Differential AS events were defined as $\mathrm{p}<0.05$.

\section{Identification of Survival Associated AS Events and Construction AS Related Prognostic Signature}

The AS events were visualized by Upset plot using UpSetR package ( $\mathrm{R}$ version 4.0.3). Survival associated AS events were identified by univariate Cox regression using $\mathrm{R}$ software. AS events with $\mathrm{P}<0.05$ were used in further research. Lasso regression was performed to remove AS events having high correlation with each other. Multivariate Cox regression was performed to determine the prognostic value of each AS event. Finally, the prognostic signature model was constructed: Risksc ore $=\sum_{i}^{n} P S I i * \beta i$ ( $\beta$ represents the regression coefficient of each event).

\section{Prognostic Signature Validation}

Based on risk score, patients with cholangiocarcinoma were divided into two groups (high/low risk). K-M survival curve and Log-Rank tests were applied to compare overall survival (OS) between high and low risk groups. The ROC curve was applied to validate the predictive effect of the prognostic signature by calculating 5 -year survival in $\mathrm{R} 4.0 .3$. Univariate 


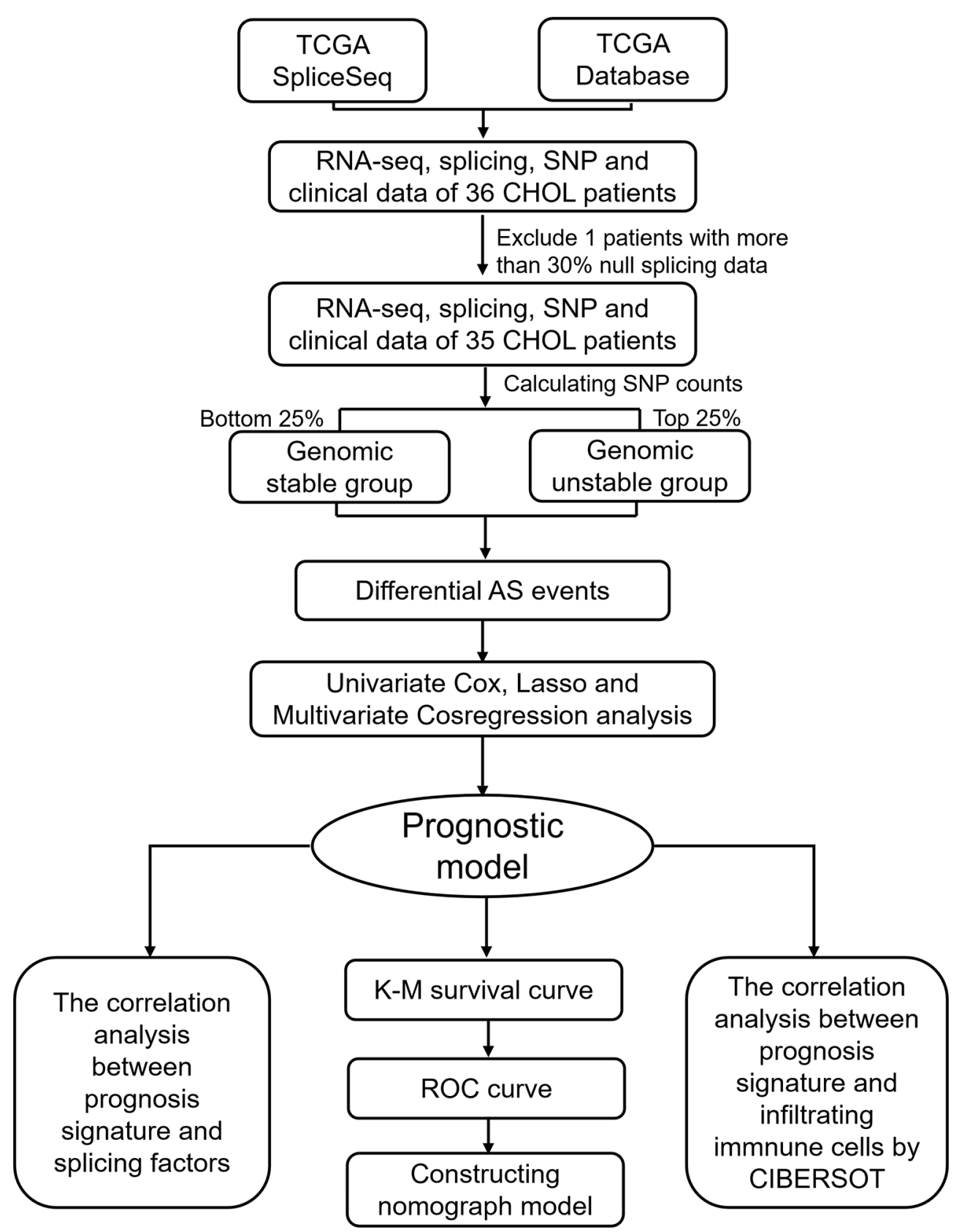

FIGURE 1 | Flow diagram of the approach used in this study.

Cox regression and Multivariate Cox regression were applied to calculate the hazard ratio (HR) of the high-risk score in OS.

\section{Correlation Between Splicing Factors and Survival Associated AS Events}

Information about 404 splicing factors was obtained from a previous study (15). The expression of splicing factors was obtained from TCGA database. Pearson correlation analysis was performed to assess the relationship between splicing factor expression and the PSI value of AS events. Splicing factors and AS events with $\mathrm{P}<0.05$ and correlation coefficient $>0.7$ were selected for building correlation plots with Cytoscape 3.7.2.

\section{Immune Cell Infiltration Analysis}

CIBERSORT algorithm (http://cibersort.stanford.edu/), a computational framework providing immune cell type information from RNA profiles (16), was used to analyze the 
infiltering immune cells in cholangiocarcinoma tissue. Twelve cases with CIBERSORT P values were selected for the further analysis. These cases were divided into high-risk $(n=4)$ and lowrisk $(n=8)$ groups based on their risk scores. The differential immune cell types between high- and low-risk groups were identified using the vioplot package of $\mathrm{R}$ 4.0.3.

\section{RESULTS}

\section{Clinical Characteristics and Integrated AS Events in Patients With Cholangiocarcinoma}

The workflow of this study is shown in Figure 1. In total, 36 patients with cholangiocarcinoma were enrolled in this study from TCGA. The baseline characteristics of enrolled patients are listed in Table 1. We identified 2146 alternate acceptor (AA) events in 1639 genes, 1846 alternate donor (AD) events in 1406 genes, 4877 alternate promoter (AP) events in 2700 genes, 5204 alternate termination (AT) events in 2965 genes, 9480 exon skipping (ES) events in 4768 genes, 105 mutually exclusive exon (ME) events in 103 genes, and 1856 retained intron (RI) in 1303 genes (Figure 2A).

\section{Identification of Genomic Instability Related AS Events in Patients With Cholangiocarcinoma}

To identify genomic instability related AS events, the cumulative quantities of somatic mutations in each patient were calculated and sorted in descending order. The top 25\% $(n=9)$ and bottom $25 \%(n=9)$ of patients were assigned to GU-like and GS-like

TABLE 1 | Characteristics of patients with cholangiocarcinoma from TCGA database.

\begin{tabular}{lcc}
\hline Characteristics & No. of patients & $\%$ \\
\hline Age & & 100.00 \\
$\geq 70$ & 15 & 41.67 \\
$<70$ & 21 & 58.33 \\
Sex & & 100.00 \\
Female & 20 & 57.14 \\
Male & 26 & 74.29 \\
Stage & & 100.00 \\
I & 19 & 54.29 \\
II & 9 & 25.71 \\
III & 1 & 2.86 \\
IV & 7 & 20.00 \\
T category & & 100.00 \\
T1 & 19 & 54.29 \\
T2 & 12 & 34.29 \\
T3 & 5 & 14.29 \\
N category & & 100.00 \\
N0 & 26 & 74.29 \\
N1 & 5 & 14.29 \\
unknown & 5 & 14.29 \\
M category & & 100.00 \\
M0 & 28 & 80.00 \\
M1 & 5 & 14.29 \\
unknown & 3 & 8.57 \\
\end{tabular}

groups, respectively. Then the AS events in patients in GU-like and GS-like groups were compared to identify differential AS events. In total, 644 differential AS events, with $\mathrm{P}$ values $<0.05$, were identified. A heat map of the top 40 differential AS events was constructed (Figure 2B). Genes involved in the differential AS events were enriched 10 Gene Ontology (GO) and 25 KEGG pathways (Figures 2C, D).

\section{Construction of Survival-Associated AS Prognostic Model}

Univariate cox regression analysis with $\mathrm{P}<0.05$ identified 26 AS events associated with cholangiocarcinoma progression (Figures 3A, B). Lasso regression analysis was performed on the 26 OSrelated AS events to identify the events highly associated with cholangiocarcinoma (Figures 3C, D). Multivariate cox regression was applied to identify independent prognostic AS events. Finally, six AS events, SLC38A10-44114-AT, IL18BP17488-RI, NBPF10-5531-ES, THNSL2-54469-ME, FAM3A90629-ES, and KIAA1432-85794-AT, were identified as independent risk factors for OS in cholangiocarcinoma (Figure 3E). The risk score of each AS event was calculated (Table 2).

\section{Validation of the Prognostic Signature in Patients With Cholangiocarcinoma}

We validated the predictive capability and efficiency of the prognostic signature. The risk score distribution curve showed that patients with cholangiocarcinoma and higher risk score have shorter survival time (Figures 4A, B). K-M survival curve analysis verified that patients with higher risk scores had poorer OS, $\mathrm{P}<0.05$ (Figure 4C). ROC curve (AUC $=0.981$ ) analysis was performed to validate the efficiency of the risk score in OS prediction (Figure 4D) and the univariate and multivariate Cox regression HR values for OS were 2.026 and 2.362, respectively (Figures $\mathbf{5 A}, \mathbf{B}$ ). Collectively, these data demonstrate that the risk score of cancer related AS can be used to predict OS in patients with cholangiocarcinoma. In addition, we constructed a nomograph model predicting 1-, 3-, and 5-year survival of patients with cholangiocarcinoma (Figure 6).

\section{The Splicing Factors Regulating the Prognostic AS Events}

Four AS events and 18 splicing factors were identified using a Pearson's correlation $\mathrm{R}$ value of $>0.7$ and univariate cox regression $\mathrm{P}$ value of $<0.05$ (Figure 7). Among these, SLC46A1-39899-ES, IARS-86836-ES, and ALDH1A3-32741AT are upregulated AS events. The remained CDK10-38118ES is a downregulated event.

HNRNPC is a core splicing factor that is positively correlated with down-regulated AS events. CCDC12, CLASRP, CLK4, RBM5, SEC31B, SRSF5, CIRBP, SNRNP70, ZRSR2, PPWD1, CLK1, CDK11A, NOSIP, U2AF1L4, RBM26, HNRNPC, HSPA1B, and CELF2 are core splicing factors that are negatively correlated with up regulated AS. We examined the 

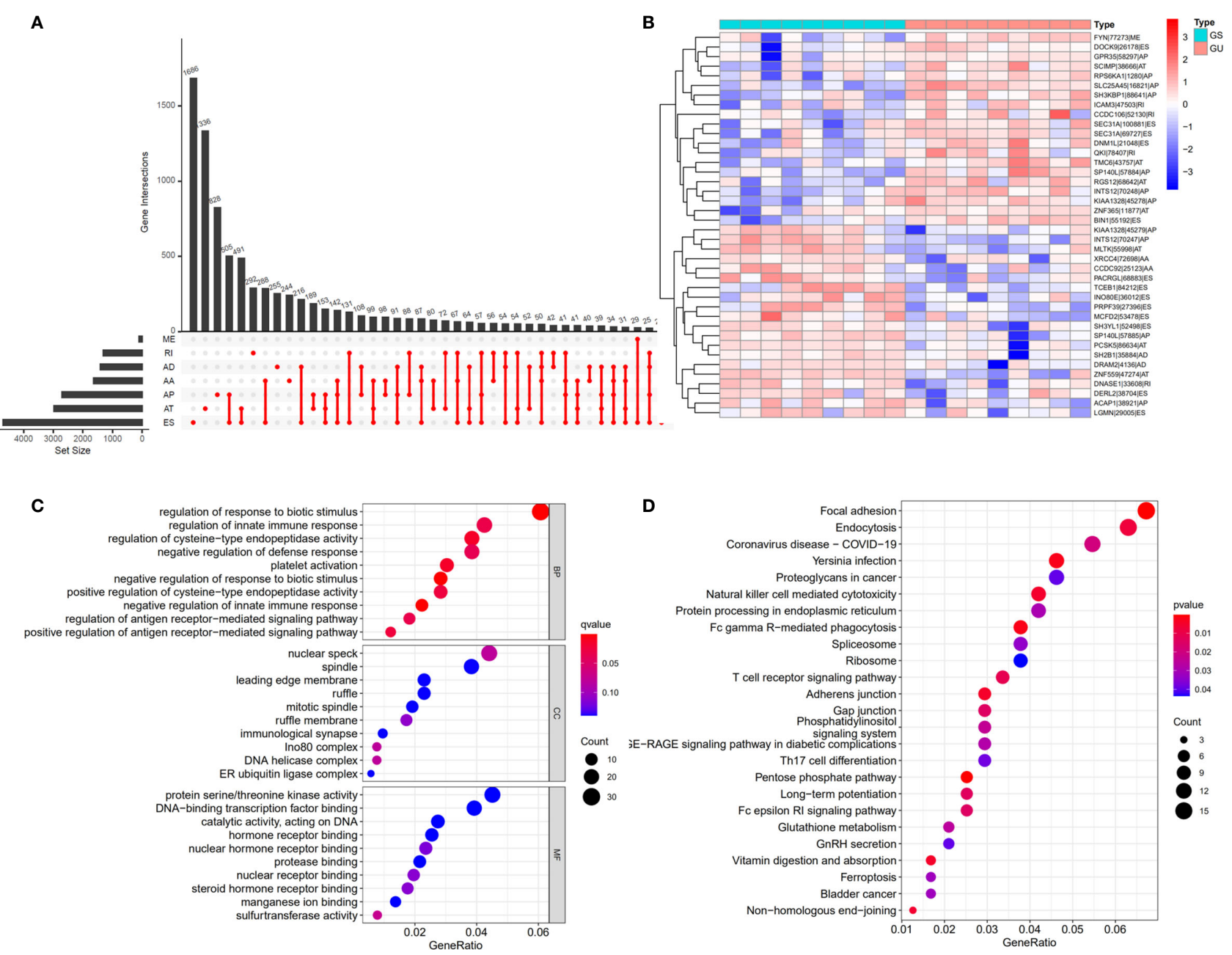

FIGURE 2 | Differential alternative splicing (AS) events between genomic stable (GS-like) and unstable (GU-like) patients. (A) AS events and related genes in cholangiocarcinoma patients. (B) Differential AS events between GU-like and GS-like groups. (C) Bubble graph showing GO analysis of differential AS events. (D) Bubble graph showing KEGG analysis of AS events.

relationship between these splicing factors and prognosis in patients with cholangiocarcinoma. Patients with higher CDK11A expression levels had higher disease-free survival rates $(\mathrm{P}=0.023)$ than did patients with lower $\mathrm{CDK} 11 \mathrm{~A}$ expression levels. Patients with higher CIRBP expression levels had higher OS, but the associated $\mathrm{P}$ value is approaching insignificance ( $\mathrm{P}=0.095$, Figure 8).

\section{Revealing the Relationship Between Prognostic Signature and Tumor- Infiltrating Immune Cells in Tumor Microenvironment}

The tumor-infiltrating immune cells were identified with CIBERSORT. 12 patients were enrolled in this study with the $\mathrm{P}$ value of CIBERSORT $<0.05$. The infiltrated immunes cells in cholangiocarcinoma are shown in Figure 9A. Among 22 kinds of immune cells, M2 macrophage are the main cell types that infiltrate in the cholangiocarcinoma tissue. Compared with low prognostic signature patients, high prognostic signature patients exhibited higher proportion of CD8+ T cells (Figures 9B, C). Additionally, the expression of PD-L1 is upregulated in patients with high AS risk score (Figure 9D).

\section{DISCUSSION}

The increasing incidence and poor outcomes for cholangiocarcinoma mean that biomarkers for diagnosis and therapy are urgently required. To the best of our knowledge, the biomarkers widely used in the clinic, including carcinoembryonic antigens (CEAs), CA-199, CA-242, and CA- 
A

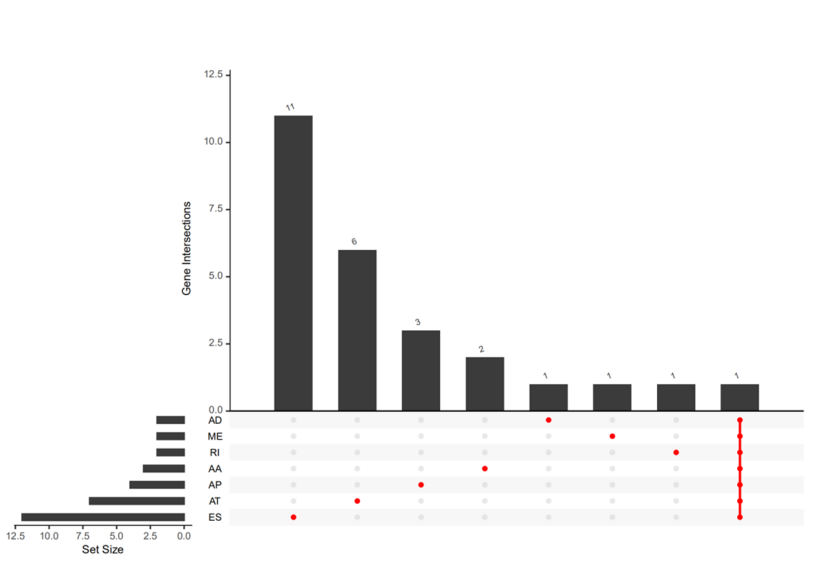

B

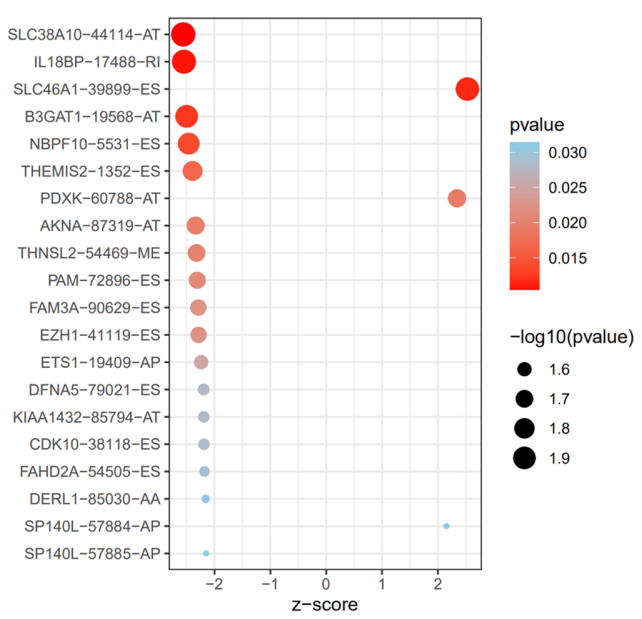

D

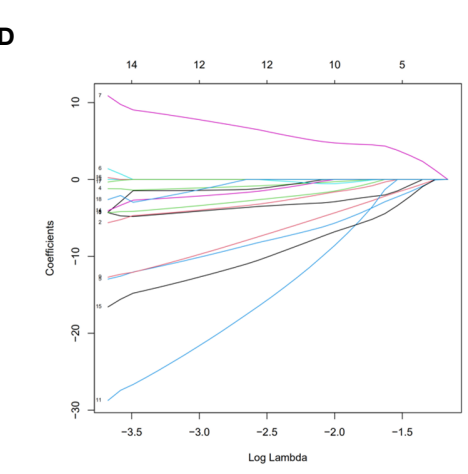

E
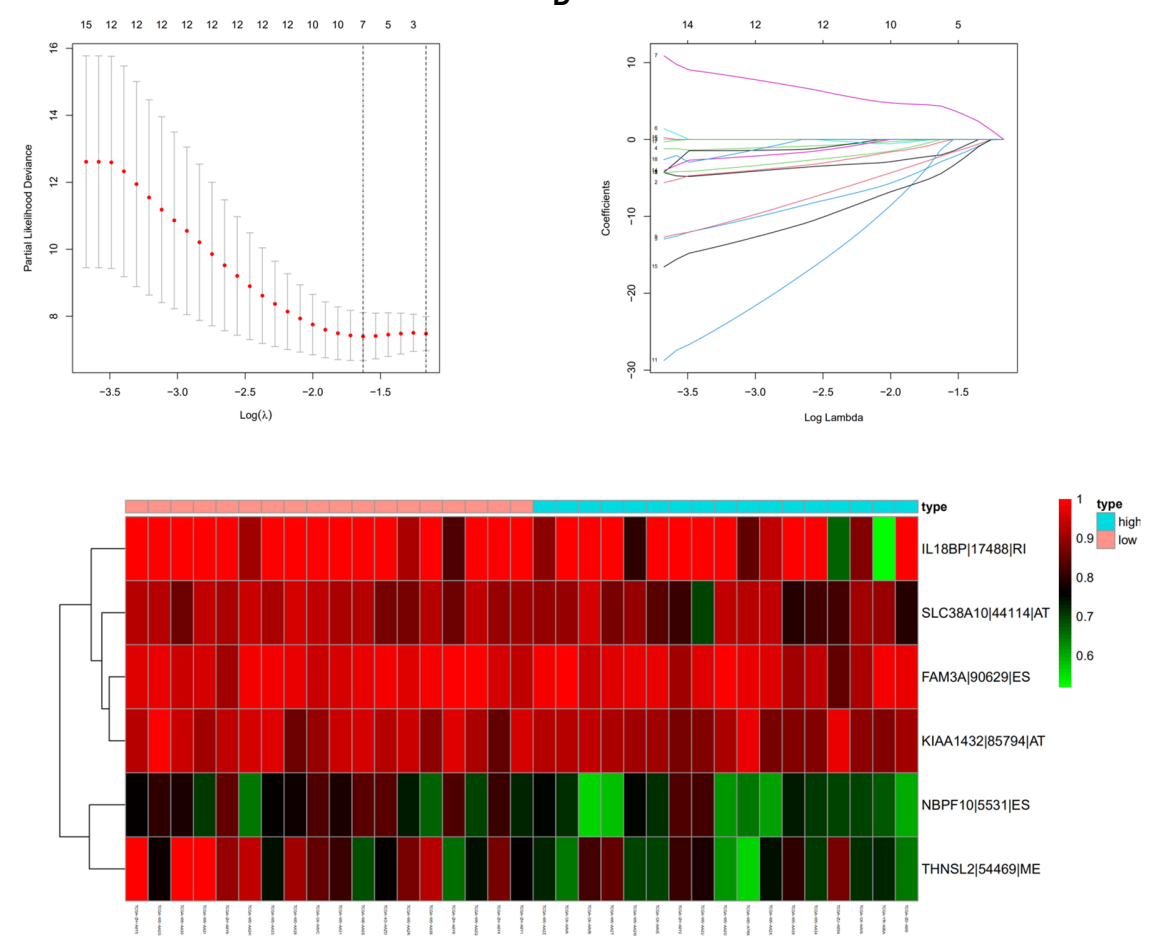

FIGURE 3 | Prognostic signature construction based on differential alternative splicing (AS) events between genomic stable (GS-like) and unstable (GU-like) groups. (A) Overall survival related AS events in patients with cholangiocarcinoma distinguished by alternate acceptor (AA), alternate donor (AD), alternate promoter (AP), alternate termination (AT), exon skipping (ES), mutually exclusive exon (ME), and retained intron (RI) splicing mode. (B) Bubble graph of overall survival related AS events in cholangiocarcinoma with $\mathrm{P}$ values and z-scores. (C, D) cvFit and lambda graph of the lasso regression model. (E) Heat map showed the expression of AS events enrolled in our prognosis model.

TABLE 2 | Multivariate cox model.

\begin{tabular}{lcccr}
\hline id & coef & HR & HR.95L & HR.95H \\
\hline SLC38A10-44114-AT & -15.32 & $2.23 \mathrm{E}-07$ & $1.61 \mathrm{E}-12$ & 0.031 \\
IL18BP-17488-RI & -6.09 & 0.002 & $1.23 \mathrm{E}-05$ & 0.422 \\
NBPF10-5531-ES & -17.24 & $3.24 \mathrm{E}-08$ & $2.23 \mathrm{E}-12$ & 0 \\
THNSL2-54469-ME & -15.04 & $2.95 \mathrm{E}-07$ & $3.19 \mathrm{E}-11$ & 0.011 \\
FAM3A-90629-ES & -33.3 & $3.47 \mathrm{E}-15$ & $3.14 \mathrm{E}-24$ & 0.003 \\
KIAA1432-85794-AT & -29.78 & $1.16 \mathrm{E}-13$ & $2.78 \mathrm{E}-23$ & 0 \\
\end{tabular}


A

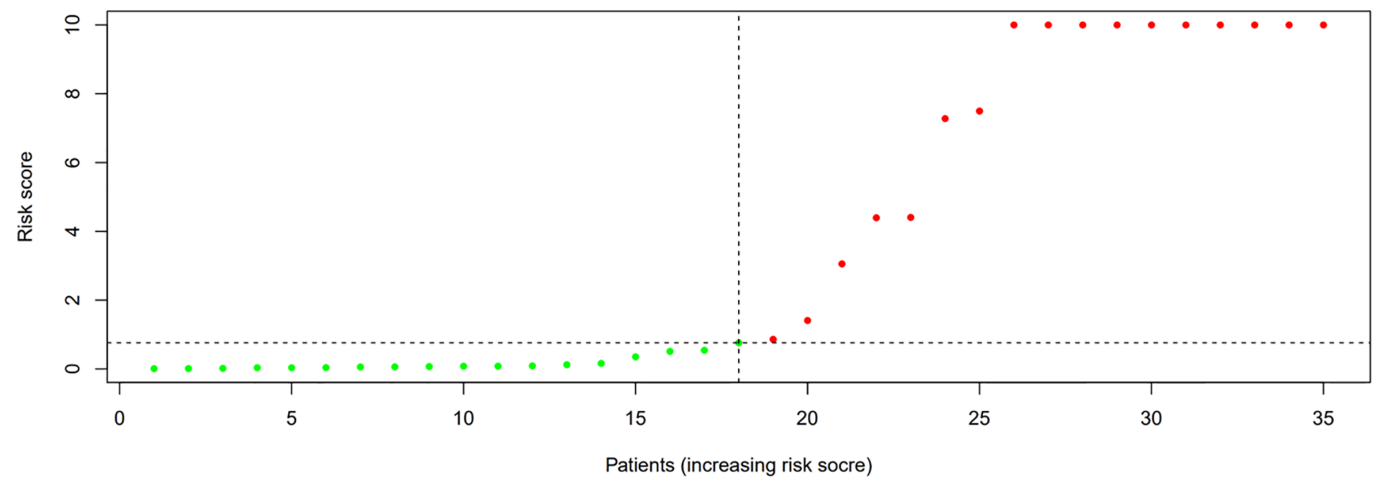

B

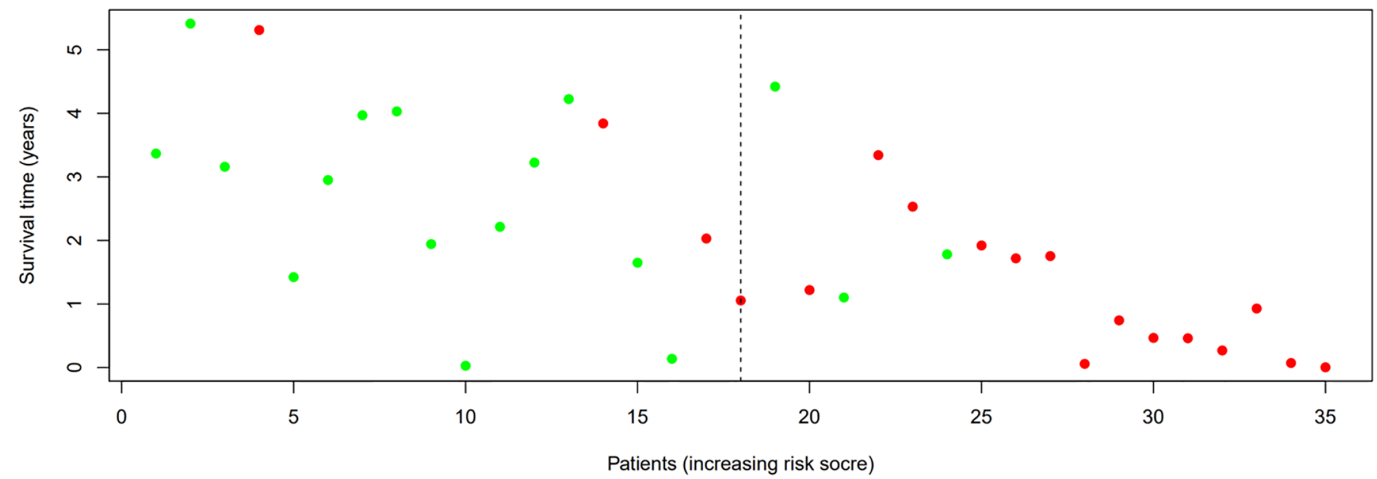

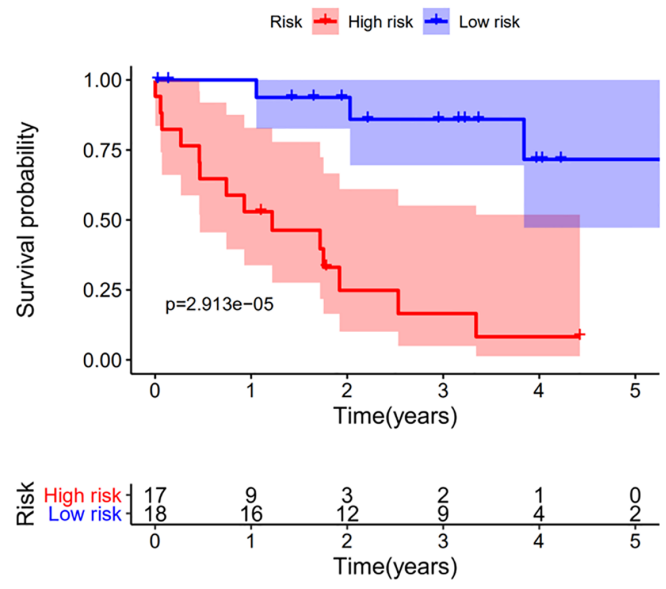

ROC curve $($ AUC $=0.981)$

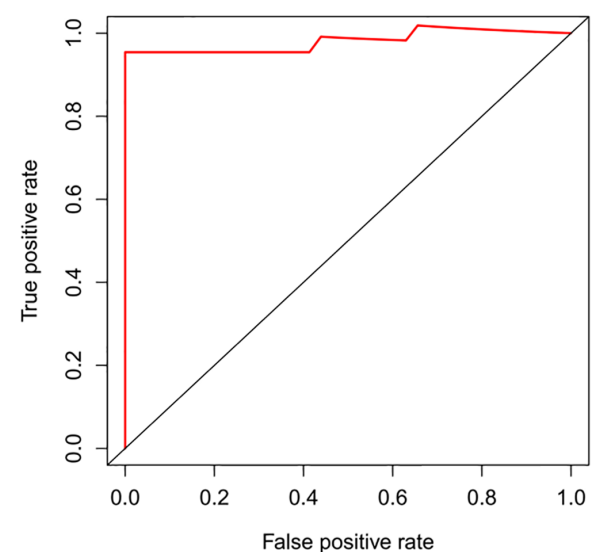

FIGURE 4 | Prognostic model validation. (A, B) Survival time and survival status of patients with different risk scores. (C) Survival curve of patients with high and low risk scores. (D) Receiver operating characteristic curve of the prognostic model.

50, have limited cholangiocarcinoma diagnostic and prognostic sensitivity and specify. The technical developments in sequencing techniques, have led to the wide clinical application of genetic diagnosis. Recently, transcriptome signatures have been applied to predict the outcome of cholangiocarcinoma. Wada $\mathrm{Y}$ and colleagues constructed a model based on 8 gene expression (BIRC5, CDC20, CDH2, CENPW, JPH1, MAD2L1, NEIL3, and
POC1A), which predicts the recurrence of cholangiocarcinoma with AUC of ROC $=0.92$ (17). Xiaozai Xie and colleagues constructed a model predicting the overall survival of cholangiocarcinoma (AUC of ROC $=0.938$ ) based on 5 lncRNA expression (18). In this study, we additionally provide an effective model based on genomic instability-related AS events for predicting OS with AUC of ROC curve of 0.981 . 

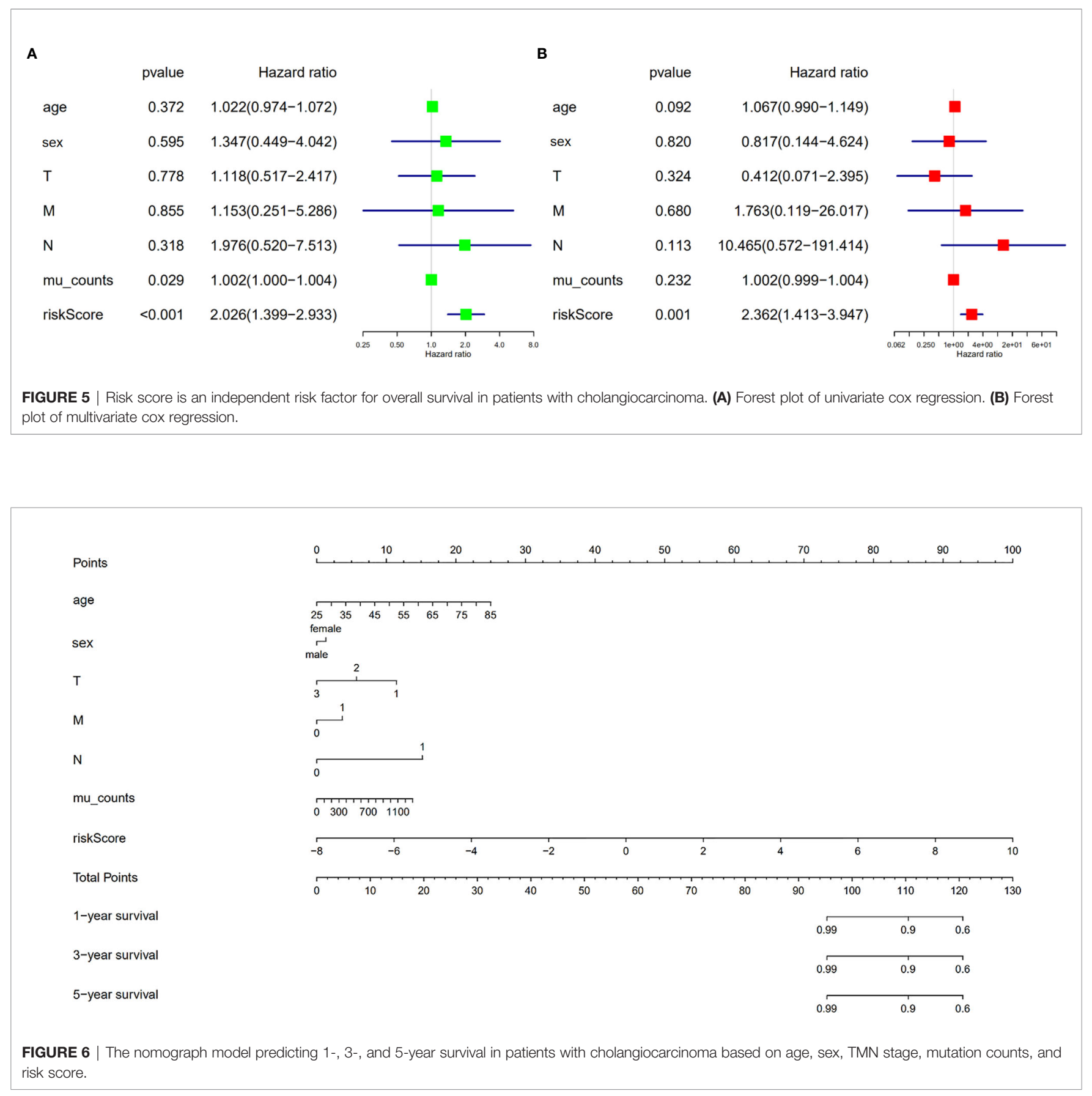

Genomic instability has been recognized a hallmark of carcinoma genesis. Recent studies have paid attentions to the role of genomic instability in the progression and recurrence indicating that the degree of genomic instability has prognostic implication. Although the molecular mechanisms of genomic instability remain unclear, previous studies have revealed that alternative splicing (AS) are associated with genomic instability (19). Some formula based on alternative splicing signature have been applied to quantify genomic instability degree (20). Recent studies have focused on the AS network, leading to the construction of prognostic signature models based on comprehensive AS events which suitable levels of predictivity and efficiency for carcinoma prognosis (21-23). Whether genomic instability-related AS events could effectively predict prognosis of cholangiocarcinoma remains unclear.

In the present study, we obtained single nucleotide polymorphism data of patients with cholangiocarcinoma from TCGA data sets. We identified differential AS events by comparing patients with genomic stability and those with genomic instability. Then, Univariate Cox regression analysis 

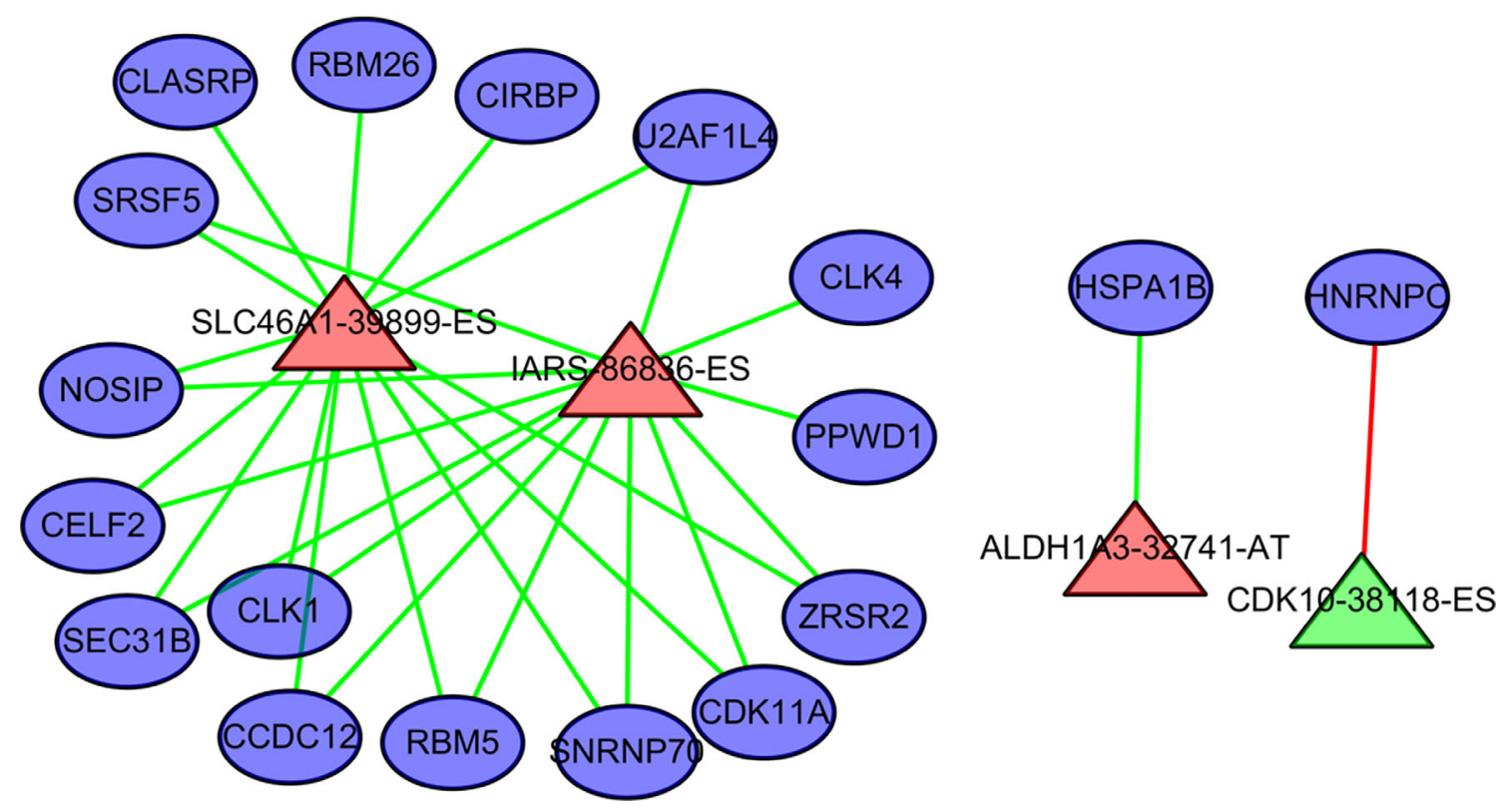

FIGURE 7 | The regulation relationship between alternative splicing (AS) events and splicing factors. The ovals represent splicing factors. The red triangles represent AS events associated with poor outcome in cholangiocarcinoma. Green triangles represent AS events negatively associated with poor outcome in cholangiocarcinoma. The lines between AS events and splicing factors represent the relationship between them. Red lines represent upregulation. Green lines represent downregulation.

revealed 26 differential AS events that were associated with the OS in cholangiocarcinoma. K-M survival and ROC analyses showed that this model has robust sensitivity and specify for predicting OS in patients with cholangiocarcinoma. However, a study using a larger cohort is needed to verify the efficiency of our model.

In our prognostic model, we identified the key roles of SLC46A1 and IARS AS in predicting the OS in patients with cholangiocarcinoma. SLC46A1 belongs to solute carrier family and participates in the import of heme folate. Previous studies show that SLC46A1 is abundant in the liver and is responsible for iron metabolism (24). Consistent with our results, Hlavac and colleagues found that SLC46A1 variants are associated with ERBB2/HER2 status and disease-free survival in hormonally treated patients with breast carcinoma (25). The underlying mechanism by which SLC46A1 variants affect cholangiocarcinoma prognosis requires further research.

Isoleucine-tRNA synthetase (IARS) is responsible for aminoacyl tRNA biosynthesis, which plays an essential role in protein translation. Recently, the IARS deficiency has been associated with human disease $(26,27)$. Hsu and colleagues found that IARS expression is upregulated in oral cavity squamous cell carcinoma (28). Additionally, our results show that IARS-86836-ES variants are associated with poor OS in patients with cholangiocarcinoma. The mechanism underlying this may be associated with insufficient aminoacylation activity to meet translational demand in tumor cells (29).

We then tried to explore the upstream regulators of prognosis associated AS events. Differential expression and hotspot mutations of splicing factor genes have recently been reported in numerous malignancies, suggesting the importance of splicing factors in cancer development and progression. Pearson correlation analysis revealed that CCDC12, CLASRP, CLK4, RBM5, SEC31B, SRSF5, CIRBP, SNRNP70, ZRSR2, PPWD1, CLK1, CDK11A, NOSIP, U2AF1L4, RBM26, HNRNPC, HSPA1B, and CELF2 negatively regulate prognosis associated AS events. Additionally, patients with cholangiocarcinoma were divided into high- and low-expression groups based on splicing factor expression. K-M survival curve analysis revealed that patients with high CDK11A expression levels had higher disease-free survival rates. Consistently, previously studies have reported the tumor-promoting anti-cancer effects of CDK11A $(30,31)$. Liu and colleagues also found that CDK11A upregulation suppresses cellular proliferation by inducing cell cycle arrest (32).

Recently, immune therapy has emerged as a promising treatment strategy for solid tumors. We summarized previous reported transcriptome signatures related to the change of immune microenvironment in cholangiocarcinoma (Supplement Table 1). Michele Ghidini and colleagues have revealed the characterization of the immune-related transcriptome in cholangiocarcinoma (33). They found that high CTLA4 expression, representing the enrichment of Treg cells, in adjacent tissue is associated with the poor recurrence free survival of cholangiocarcinoma. In addition to their study, we analyzed difference of infiltrated immune cells in patients with different alternative splicing signature. We found that the proportion of $\mathrm{CD} 8+\mathrm{T}$ cells is upregulated in carcinoma tissue of 


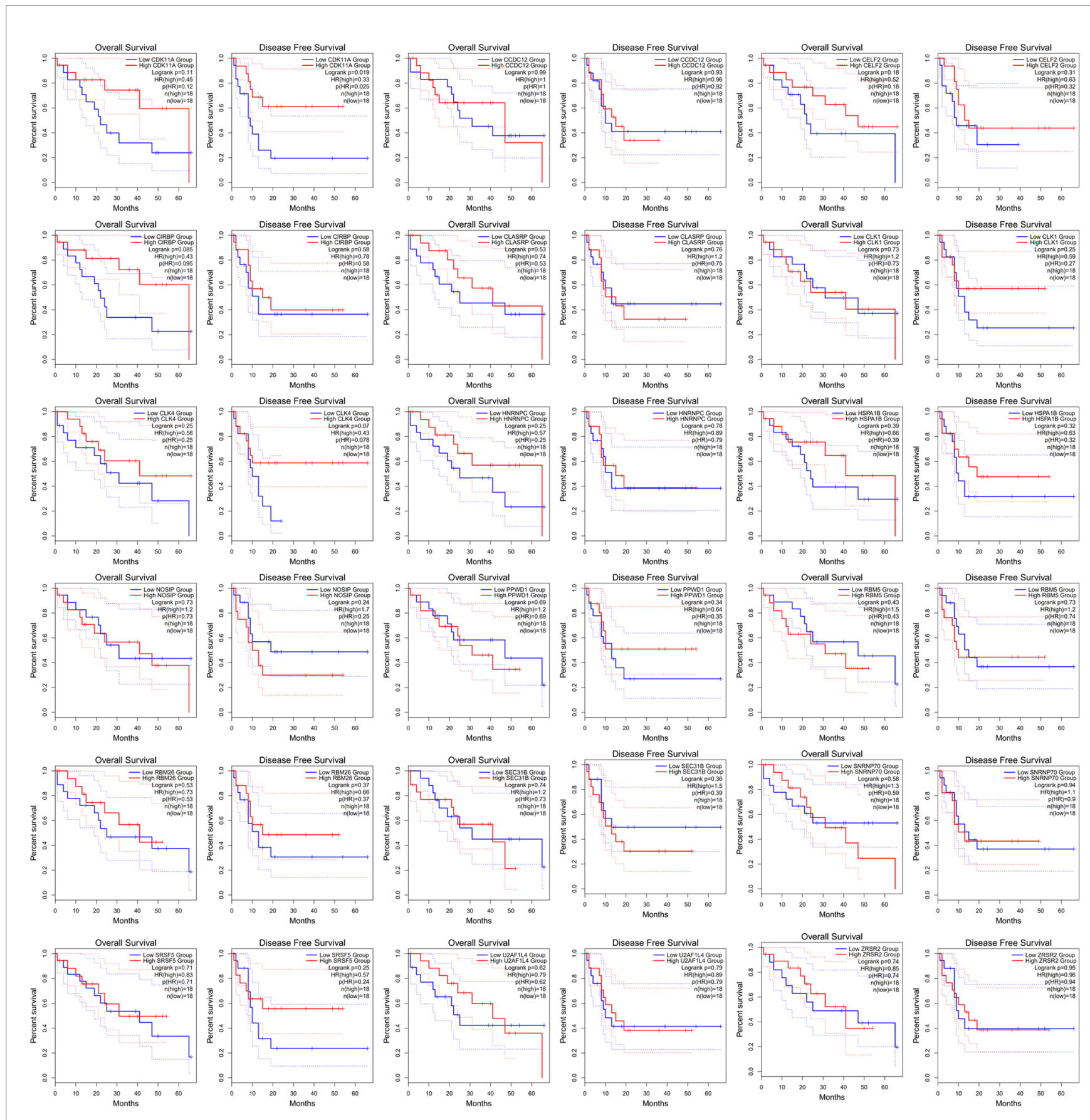

FIGURE 8 | The receiver operating characteristic curve of alternative splicing (AS) signature related splicing factors in overall survival and disease-free survival in patients with cholangiocarcinoma.

patients with higher splicing signature scores. Ying Zhu and colleagues found that INF- $\gamma$ secretion by CD8+ T cells may increase cancer cell PD-L1 expression (34). Upregulated PD-L1 on cancer cells has been recognized as a marker of immune escape and poor outcome in patients with cholangiocarcinoma (35). Consistent with this, we found higher levels of PD-L1 expression in the high-risk group. Therefore, the high-risk group may more effectively respond to anti-PD-L1/PD-1 therapy. Finally, we also produced a nomograph model for predicting 1-, 3-, and 5-year survival in patients with cholangiocarcinoma based on age, sex, TMN stage, mutation counts, and risk score.

In conclusion, we have developed a prognostic signature for OS in patients with cholangiocarcinoma based on cancer 
A

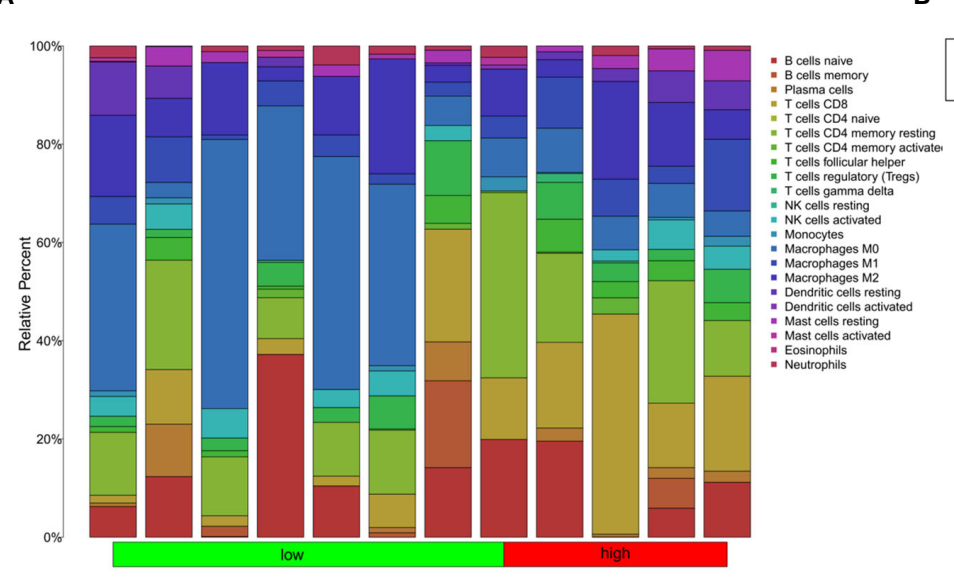

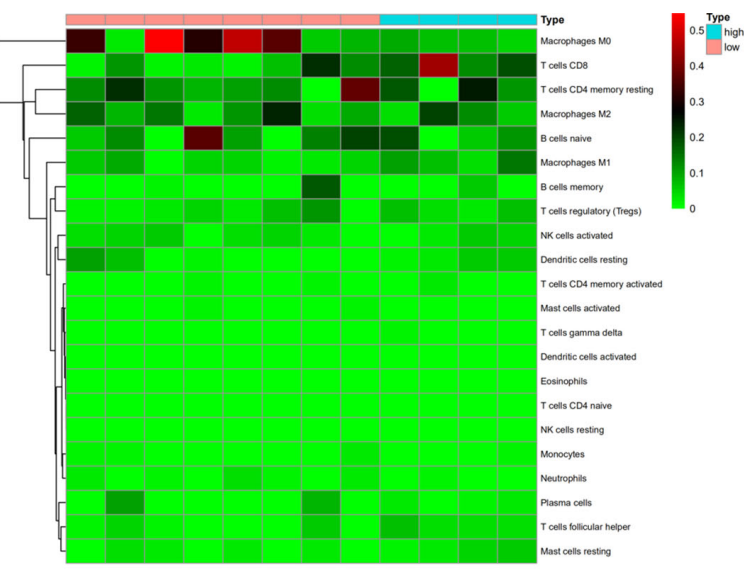

C

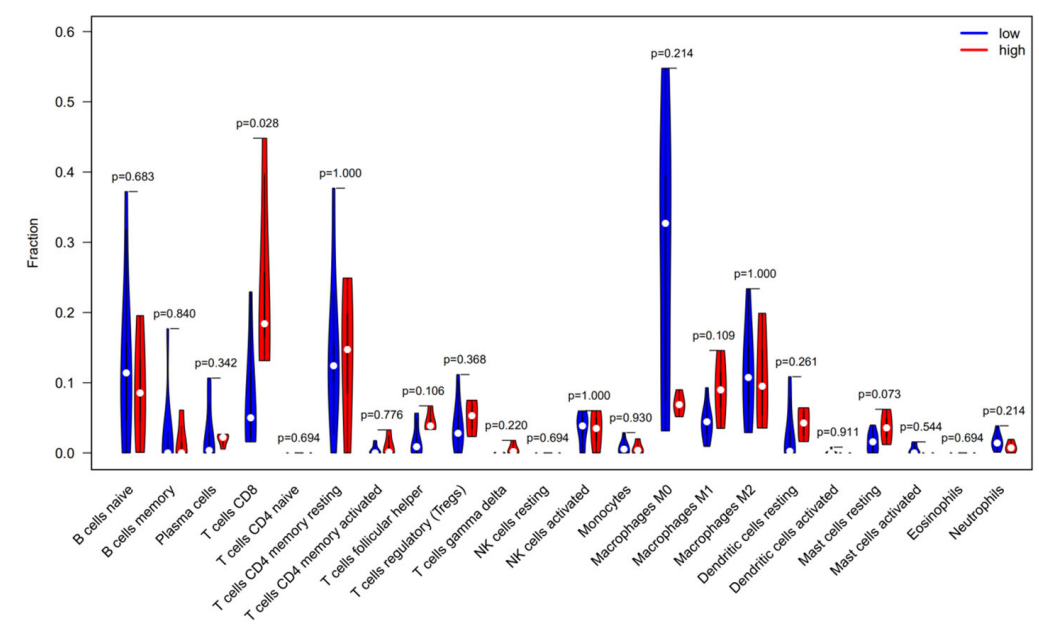

D

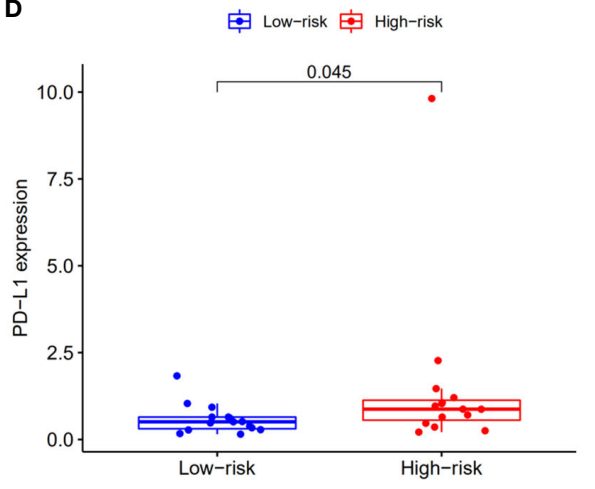

FIGURE 9 | Infiltrating immune cells in cholangiocarcinoma. (A) Bar plot showing the proportion of 22 kinds of immune cells in patients with CIBERSORT value $<0.05$. (B) Heat map showing the proportion of 22 types of immune cells in high- and low-risk groups. (C) Vioplot showing the compression of each immune cell type between high- and low-risk groups. (D) Bar plot of PD-L1 expression in high- and low-risk groups.

pathway-related AS events. Additionally, AS events SLC46A139899-ES, IARS-86836-ES, and the CDK11A splicing factor may be therapeutic targets for cholangiocarcinoma. Anti-PDL1/PD-1 immunotherapy may be a promising therapeutic strategy for patients with cholangiocarcinoma and high-risk scores. However, the small sample size used in this study means that our results require further external examination.

\section{DATA AVAILABILITY STATEMENT}

The datasets presented in this study can be found in online repositories. The names of the repository/repositories and accession number(s) can be found in the article/supplementary material.

\section{AUTHOR CONTRIBUTIONS}

ZZ designed the study and wrote the manuscript. ZL collected data and completed the data analysis. GZ and JH wrote the R script. JG and LZ guided the study and checked the data. All authors contributed to the article and approved the submitted version.

\section{FUNDING}

This study was funded by the National Natural Science Foundation of China (Grant NO.81970510), Talent Project of Chongqing (CQYC2019050790), and the Graduate Student Innovation Project of Chongqing (Grant number: CYB20153). 


\section{ACKNOWLEDGMENTS}

We acknowledge the TCGA and TCGAspliceSeq for the transcriptomics, simple nucleotide variation, clinical phenotype, and splicing data. We acknowledge Chan Qiu for his assistance in writing the R script.

\section{REFERENCES}

1. Munoz-Garrido P, Rodrigues PM. The Jigsaw of Dual HepatocellularIntrahepatic Cholangiocarcinoma Tumours. Nat Rev Gastroenterol Hepatol (2019) 16(11):653-5. doi: 10.1038/s41575-019-0185-z

2. Banales JM, Marin JJG, Lamarca A, Rodrigues PM, Khan SA, Roberts LR, et al. Cholangiocarcinoma 2020: The Next Horizon in Mechanisms and Management. Nat Rev Gastroenterol Hepatol (2020) 17(9):557-88. doi: 10.1038/s41575-020-0310-z

3. Bertuccio P, Malvezzi M, Carioli G, Hashim D, Boffetta P, El-Serag HB, et al. Global Trends in Mortality From Intrahepatic and Extrahepatic Cholangiocarcinoma. J Hepatol (2019) 71(1):104-14. doi: 10.1016/j.jhep.2019.03.013

4. Negrini S, Gorgoulis VG, Halazonetis TD. Genomic Instability-an Evolving Hallmark of Cancer. Nat Rev Mol Cell Biol (2010) 11(3):220-8. doi: 10.1038/nrm2858

5. Suzuki K, Ohnami S, Tanabe C, Sasaki H, Yasuda J, Katai H, et al. The Genomic Damage Estimated by Arbitrarily Primed PCR DNA Fingerprinting is Useful for the Prognosis of Gastric Cancer. Gastroenterol (2003) 125 (5):1330-40. doi: 10.1016/j.gastro.2003.07.006

6. Wang Y, Hong Y, Li M, Long J, Zhao YP, Zhang JX, et al. Mutation Inactivation of Nijmegen Breakage Syndrome Gene (NBS1) in Hepatocellular Carcinoma and Intrahepatic Cholangiocarcinoma. PloS One (2013) 8(12):e82426. doi: 10.1371/journal.pone.0082426

7. Bao S, Zhao H, Yuan J, Fan D, Zhang Z, Su J, et al. Computational Identification of Mutator-Derived lncRNA Signatures of Genome Instability for Improving the Clinical Outcome of Cancers: A Case Study in Breast Cancer. Briefings Bioinf (2020) 21(5):1742-55. doi: 10.1093/bib/bbz118

8. Blencowe BJ. Alternative Splicing: New Insights From Global Analyses. Cell (2006) 126(1):37-47. doi: 10.1016/j.cell.2006.06.023

9. Sebestyen E, Zawisza M, Eyras E. Detection of Recurrent Alternative Splicing Switches in Tumor Samples Reveals Novel Signatures of Cancer. Nucleic Acids Res (2015) 43(3):1345-56. doi: 10.1093/nar/gku1392

10. Thanee M, Loilome W, Techasen A, Sugihara E, Okazaki S, Abe S, et al. CD44 Variant-Dependent Redox Status Regulation in Liver Fluke-Associated Cholangiocarcinoma: A Target for Cholangiocarcinoma Treatment. Cancer Sci (2016) 107(7):991-1000. doi: 10.1111/cas.12967

11. Yosudjai J, Wongkham S, Jirawatnotai S, Kaewkong W. Aberrant mRNA Splicing Generates Oncogenic RNA Isoforms and Contributes to the Development and Progression of Cholangiocarcinoma. Biomed Rep (2019) 10(3):147-55. doi: 10.3892/br.2019.1188

12. Yao J, Caballero OL, Huang Y, Lin C, Rimoldi D, Behren A, et al. Altered Expression and Splicing of ESRP1 in Malignant Melanoma Correlates With Epithelial-Mesenchymal Status and Tumor-Associated Immune Cytolytic Activity. Cancer Immunol Res (2016) 4(6):552-61. doi: 10.1158/23266066.CIR-15-0255

13. Kim EK, Yoon SO, Jung WY, Lee H, Kang Y, Jang YJ, et al. Implications of NOVA1 Suppression Within the Microenvironment of Gastric Cancer: Association With Immune Cell Dysregulation. Gastric Cancer Off J Int Gastric Cancer Assoc Japanese Gastric Cancer Assoc (2017) 20(3):438-47. doi: 10.1007/s10120-016-0623-3

14. Tan DSW, Chong FT, Leong HS, Toh SY, Lau DP, Kwang XL, et al. Long Noncoding RNA Egfr-AS1 Mediates Epidermal Growth Factor Receptor Addiction and Modulates Treatment Response in Squamous Cell Carcinoma. Nat Med (2017) 23(10):1167-75. doi: 10.1038/nm.4401

15. Seiler M, Peng S, Agrawal AA, Palacino J, Teng T, Zhu P, et al. Somatic Mutational Landscape of Splicing Factor Genes and Their Functional Consequences Across 33 Cancer Types. Cell Rep (2018) 23(1):282-96.e4. doi: 10.1016/j.celrep.2018.01.088

16. Newman AM, Liu CL, Green MR, Gentles AJ, Feng W, Xu Y, et al. Robust Enumeration of Cell Subsets From Tissue Expression Profiles. Nat Methods (2015) 12(5):453-7. doi: 10.1038/nmeth.3337

\section{SUPPLEMENTARY MATERIAL}

The Supplementary Material for this article can be found online at: https://www.frontiersin.org/articles/10.3389/fonc.2021. 666847/full\#supplementary-material

17. Wada Y, Shimada M, Yamamura K, Toshima T, Banwait JK, Morine Y, et al. A Transcriptomic Signature for Risk-Stratification and Recurrence Prediction in Intrahepatic Cholangiocarcinoma. Hepatol (2021). doi: 10.1002/hep.31803

18. Xie X, Wang Y, Zhang S, Li J, Yu Z, Ding X, et al. A Novel five-lncRNA Signature Panel Improves High-Risk Survival Prediction in Patients With Cholangiocarcinoma. Aging (2021) 13(2):2959-81. doi: 10.18632/aging.202446

19. Shkreta L, Chabot B. The RNA Splicing Response to DNA Damage. Biomolecules (2015) 5(4):2935-77. doi: 10.3390/biom5042935

20. Cahill K. Alternative Splicing and Genomic Stability. Phys Biol (2004) 1(1-2): C1-4. doi: 10.1088/1478-3967/1/2/C01

21. Liu Y, Jia W, Li J, Zhu H, Yu J. Identification of Survival-Associated Alternative Splicing Signatures in Lung Squamous Cell Carcinoma. Front Oncol (2020) 10:587343. doi: 10.3389/fonc.2020.587343

22. Suh YS, Na D, Lee JS, Chae J, Kim E, Jang G, et al. Comprehensive Molecular Characterization of Adenocarcinoma of the Gastroesophageal Junction Between Esophageal and Gastric Adenocarcinomas. Ann Surg (2020). doi: 10.1097/SLA.0000000000004303

23. Zhao Z, Li GZ, Liu YQ, Huang RY, Wang KY, Jiang HY, et al. Characterization and Prognostic Significance of Alternative Splicing Events in Lower-Grade Diffuse Gliomas. J Cell Mol Med (2020) 24(22):13171-80. doi: 10.1111/jcmm.15924

24. Li H, Wang D, Wu H, Shen H, Lv D, Zhang Y, et al. SLC46A1 Contributes to Hepatic Iron Metabolism by Importing Heme in Hepatocytes. Metabolism: Clin Exp (2020) 110:154306. doi: 10.1016/j.metabol.2020.154306

25. Hlavac V, Vaclavikova R, Brynychova V, Dvorak P, Elsnerova K, Kozevnikovova R, et al. Slc46a1 Haplotype With Predicted Functional Impact has Prognostic Value in Breast Carcinoma. Mol diagnosis Ther (2021) 25:99-110. doi: 10.1007/s40291-020-00506-2

26. Orenstein N, Weiss K, Oprescu S, Shapira R, Kidron D, Vanagaite-Basel L, et al. Bi-Allelic IARS Mutations in a Child With Intra-Uterine Growth Retardation, Neonatal Cholestasis, and Mild Developmental Delay. Clin Genet (2017) 91(6):913-7. doi: 10.1111/cge.12930

27. Kopajtich R, Murayama K, Janecke A, Haack T, Breuer M, Knisely A, et al. Biallelic IARS Mutations Cause Growth Retardation With Prenatal Onset, Intellectual Disability, Muscular Hypotonia, and Infantile Hepatopathy. Am J Hum Genet (2016) 99(2):414-22. doi: 10.1016/j.ajhg.2016.05.027

28. Hsu C, Chang K, Huang Y, Liu H, Hsueh P, Gu P, et al. Proteomic Profiling of Paired Interstitial Fluids Reveals Dysregulated Pathways and Salivary NID1 as a Biomarker of Oral Cavity Squamous Cell Carcinoma. Mol Cell Proteomics MCP (2019) 18(10):1939-49. doi: 10.1074/mcp.RA119.001654

29. Fuchs S, Schene I, Kok G, Jansen J, Nikkels P, van Gassen K, et al. AminoacyltRNA Synthetase Deficiencies in Search of Common Themes. Genet Med Off J Am Coll Med Genet (2019) 21(2):319-30. doi: 10.1038/s41436-018-0048-y

30. Sotgia F, Fiorillo M, Lisanti MP. Hallmarks of the Cancer Cell of Origin: Comparisons With "Energetic" Cancer Stem Cells (E-Cscs). Aging (2019) 11 (3):1065-8. doi: 10.18632/aging.101822

31. Zhou QH, Deng CZ, Li ZS, Chen JP, Yao K, Huang KB, et al. Molecular Characterization and Integrative Genomic Analysis of a Panel of Newly Established Penile Cancer Cell Lines. Cell Death Dis (2018) 9(6):684. doi: 10.1038/s41419-018-0736-1

32. Liu $\mathrm{T}, \mathrm{Wu} \mathrm{Y}$, Dong $\mathrm{X}, \mathrm{Pan} \mathrm{C}, \mathrm{Du} \mathrm{G}$, Yang J, et al. Identification and Characterization of the BmCyclin L1-BmCDK11A/B Complex in Relation to Cell Cycle Regulation. Cell Cycle (Georgetown Tex) (2017) 16(9):861-8. doi: 10.1080/15384101.2017.1304339

33. Ghidini M, Cascione L, Carotenuto P, Lampis A, Trevisani F, Previdi MC, et al. Characterisation of the Immune-Related Transcriptome in Resected Biliary Tract Cancers. Eur J Cancer (2017) 86:158-65. doi: 10.1016/j.ejca.2017.09.005

34. Zhu Y, Wang X, Zhang Y, Xu D, Dong J, Zhang Z, et al. Programmed Death Ligand 1 Expression in Human Intrahepatic Cholangiocarcinoma and its Association With Prognosis and CD8 T-Cell Immune Responses. Cancer Manage Res (2018) 10:4113-23. doi: 10.2147/cmar.s172719 
35. Wu H, Wei Y, Jian M, Lu H, Song Q, Hao L, et al. Clinicopathological and Prognostic Significance of Immunoscore and PD-L1 in Intrahepatic Cholangiocarcinoma. OncoTargets Ther (2021) 14:39-51. doi: 10.2147/ott.s288982

Conflict of Interest: The authors declare that the research was conducted in the absence of any commercial or financial relationships that could be construed as a potential conflict of interest.
Copyright (c) 2021 Lin, Gong, Zhong, Hu, Cai, Zhao and Zhao. This is an open-access article distributed under the terms of the Creative Commons Attribution License (CC BY). The use, distribution or reproduction in other forums is permitted, provided the original author(s) and the copyright owner(s) are credited and that the original publication in this journal is cited, in accordance with accepted academic practice. No use, distribution or reproduction is permitted which does not comply with these terms. 